\title{
Observation on Surgical Jaundice due to Noncalculous Causes with Special References to Management and Prognosis
}

\author{
Authors \\ Dr Sucheta Panigrahi (MS), Dr Sanjay Kujur (MS), Dr Jitendra Kumar Sahu (MS) \\ Department of Surgery, VSSIMSAR, BURLA, Odisha
}

\section{Introduction}

Common causes of surgical jaundice are choledocholithiasis, biliary stricture, carcinoma head of pancreas and periampullary carcinoma, malignant obstruction at porta hepatis. Though it includes both calculous and noncalculous causes, noncalculous causes are more aggressive as in this group malignant obstruction is more common. So besides the physiological and biochemical changes of obstructive jaundice there is devastating effect of malignancy. Accurate diagnosis should be made by thorough history, complete physical examination and proper investigations. Malignant obstructive jaundice patients usually presents late and early diagnosis is rather an exception than a rule. These patients need only palliative procedure like bypass. However in cases detected early, curative surgery can be done, provided the patient is fit for surgery and surgeon is experienced. Although extensive studies have been done in developed countries on various aspects of obstructive jaundice, the observation in developing countries like ours, are a few. As our diet and living habits differ from that of Western Countries, the etiology and clinical course ought to be different from that of Western people. Since study of obstructive jaundice is scarce in India and no study has been done in Western Orissa on management and prognosis of non calculous surgical jaundice, it was thought prudent to proceed with this study.

This study is undertaken with a view

1. To derive some facts regarding the etiology and clinical presentation of noncalculous surgical jaundice.

2. To acquire some knowledge about biochemical changes and radiological findings in these diseases.

3. To study the response of preoperative treatment and effect of operative management.

4. To study the postoperative morbidity and mortality of different surgical procedures undertaken for benign and malignant causes of noncalculous surgical jaundice.

\section{Materials and Methods}

This study consisted of all patients with surgical jaundice due to non-calculous causes, admitted to the surgery ward of V.S.S. Medical College, Hospital, Burla from Nov. 2015 to Nov 2017. Cases having surgical jaundice due to calculous causes are excluded from the study.

Diagnosis of non-calculus surgical jaundice was made on the basis of clinical, laboratory and radiological criteria. The confirmation of diagnosis was made at laparotomy. 


\section{Clinical Criteria}

1) History of any or all of the following in association with jaundice.

2) Abdominal pain suggestive of biliary disease or pancreatitis.

3) Previous biliary surgery.

4) Absence of exposure to hepatitis or potential hepatotoxins and absence of hepatitis like symptoms.

\section{Physical Signs}

1. Palpable gall bladder with jaundice

2. Abdominal mass

3. Absence of stigmata of chronic liver disease.

4. High fever with chill suggestive of cholangitis

5. Hepatomegaly

\section{Laboratory Criteria}

1. Disproportionate elevation of serum alkaline phosphatase or bilirubin concentration relative to serum transaminase activity.

2. Negative serologic markers for viral hepatitis or chronic active hepatitis, primary biliary cirrhosis.

\section{Ultrasonographic Criteria}

- Visualization of dilated intrahepatic or extrahepatic biliary tree imply mechanical obstruction to biliary system.

The clues to the diagnosis of dilated extrahepatic biliary channel by Ultrasonography are:

- Common duct dilatation greater than 4 to 6 $\mathrm{mm}$ in the area of right branch of the portal vein and hepatic artery (except the cases previously undergone - Cholecystectomy where upper limit is $10 \mathrm{~mm}$ and in children and neonates where the upper limit is $4 \mathrm{~mm}$ and $1 \mathrm{~mm}$ respectively).
- Indemnification of a ventrally convex large tubular structure in the hepatoduodenal ligament region.

In all cases where surgery was decided laparotomy was done. and the cause and site of obstruction was identified. Operative procedure was done on individual basis. Curative surgery was done in benign cases or early cases of malignancy. In advanced malignancy only palliative bypass procedures were done. The patients were followed up post operatively in the ward till discharged from the hospital and subsequently. The complications they developed in the post operative period and management thereof were recorded.

The detailed history, clinical examination findings, laboratory and USG examination findings, CT scan finding, operation note and post operative results were recorded in a Performa prescribed for each case. Subsequently the data's were compiled for statistical analysis.

Altogether 25 patients with noncalculous surgical jaundice were admitted to the surgical wards, out of these 23 patients Laparotomy with some form of resection or bypass procedure was done and diagnosis was confirmed at operation. Among the rest two patients, in one patient surgery was not done because of poor general condition, advanced disease and cardiological contraindication. In the other patient only drainage of pericolic abscess was done under LA and definitive surgery was deferred because of poor general condition of the patient. In these two patients diagnosis was confirmed by FNAC. So all-25 cases were studied in detail and analyzed.

\section{Observation}

Table I Age and Sex Incidence in 25 Patients of Noncalculous Surgical Jaundice

\begin{tabular}{|c|c|c|c|}
\hline Age group in yrs & No of male patient $(\%)$ & No of female patient $(\%)$ & Total \\
\hline $10-19$ & - & $1(4 \%)$ & $1(4 \%)$ \\
$20-29$ & $1(4 \%)$ & - & $1(4 \%)$ \\
$30-39$ & $2(8 \%)$ & $1(4 \%)$ & $3(12 \%)$ \\
$40-49$ & $3(12 \%)$ & $2(8 \%)$ & $5(20 \%)$ \\
$50-59$ & $1(4 \%)$ & $3(12 \%)$ & $5(20 \%)$ \\
$60-69$ & $4(16 \%)$ & $3(12 \%)$ & $7(28 \%)$ \\
$70-79$ & $2(8 \%)$ & $1(4 \%)$ & $3(12 \%)$ \\
\hline Total & $14(56 \%)$ & $11(44 \%)$ & $25(100 \%)$ \\
\hline
\end{tabular}


Table II- Causes and Sex Incidence in 25 Patients of Noncalculous Surgical Jaundice

\begin{tabular}{|l|c|c|c|}
\hline Causes & No of male patient (\%) & No of female patient (\%) & Total \\
\hline Periampullary and Pancreatic carcinoma & $10(40 \%)$ & $6(24 \%)$ & $16(64 \%)$ \\
\hline Carcinoma of gall bladder & $2(8 \%)$ & $4(16 \%)$ & $6(24 \%)$ \\
\hline $\begin{array}{l}\text { Metastatic node at porta hepatis from ca. } \\
\text { Stomach and caecum }\end{array}$ & $2(8 \%)$ & - & $2(8 \%)$ \\
\hline Choledochal cyst & - & $1(4 \%)$ & $1(4 \%)$ \\
\hline
\end{tabular}

Table III - Age Incidence of Diffrent Causes of Noncalculous Surgical Jaundice

\begin{tabular}{|c|c|c|c|c|}
\hline Age group & $\begin{array}{c}\text { No of patients of } \\
\text { Periampullary and Pancreatic } \\
\text { carcinoma }\end{array}$ & $\begin{array}{c}\text { No of patients of } \\
\text { Carcinoma of gall } \\
\text { bladder }\end{array}$ & $\begin{array}{c}\text { No of patients of } \\
\text { Metastatic node at } \\
\text { porta hepatis }\end{array}$ & $\begin{array}{c}\text { No of patients of } \\
\text { Choledochal cyst }\end{array}$ \\
\hline $10-19$ & - & - & - & - \\
$20-29$ & $1(6.2 \%)$ & - & - & - \\
$30-39$ & $3(18.7 \%)$ & $1(16.6 \%)$ & $1(50 \%)$ & - \\
$40-49$ & $3(18.7 \%)$ & $2(33.3 \%)$ & $1(50 \%)$ & - \\
$50-59$ & $2(12.5 \%)$ & $1(16.6 \%)$ & - & - \\
$60-69$ & $6(37.5 \%)$ & $2(33.3 \%)$ & - & 1 \\
$70-79$ & $1(6.2 \%)$ & 6 & 2 & - \\
\hline Total & 16 & & & - \\
\hline
\end{tabular}

Table IV - Clinical Features in 25 Patients with Noncalculous Surgical Jaundice

\begin{tabular}{|c|c|c|}
\hline Symptoms & No of patient & Percentage \\
\hline Abdominal pain & 19 & $76 \%$ \\
\hline Pruritus & 15 & $60 \%$ \\
\hline Acholic stool & 11 & $44 \%$ \\
\hline Fever & 7 & $28 \%$ \\
\hline Bleeding & 1 & $4 \%$ \\
\hline \multicolumn{3}{|l|}{ Sign } \\
\hline Palpable gall bladder & 20 & $80 \%$ \\
\hline Hepatomegaly & 15 & $60 \%$ \\
\hline Ascites & 6 & $24 \%$ \\
\hline
\end{tabular}

Table V - Laboratory Findings in 25 Patients with Noncalculous Surgical Jaundice

\begin{tabular}{|l}
\multicolumn{1}{|c}{ Laboratory investiga } \\
\hline Serum billirubin( in $\mathrm{mg} / \mathrm{dl}$ ) \\
Serum alkaline phosphatase( in IU/L) \\
SGOT (in IU/L) \\
SGPT (in IU/L) \\
Total Serum Protien ( in $\mathrm{mg} / \mathrm{dl}$ ) \\
Prothrombin time ( in sec) \\
\hline
\end{tabular}

\begin{tabular}{|llc|}
\hline Mean & +/- & SD \\
\hline 15.24 & $+/-$ & 7.39 \\
554.96 & $+/-$ & 323.32 \\
113.88 & $+/-$ & 90.88 \\
74.92 & $+/-$ & 40.19 \\
6.92 & $+/-$ & 0.48 \\
13.28 & $+/-$ & 1.4 \\
\hline
\end{tabular}

Table VI - Ultasonographic Findings in 25 Patients with Noncalculous Surgical Jaundice

\begin{tabular}{|l|c|c|}
\hline USG Findings & No of patients & Percentage \\
\hline Dilated IHBR & 25 & $100 \%$ \\
Dilated choledochus & 21 & $84 \%$ \\
Hepatomegaly & 19 & $76 \%$ \\
Dilated gall bladder & 16 & $64 \%$ \\
Pancreatic pathology & 16 & $64 \%$ \\
Ascites & 6 & $24 \%$ \\
\hline
\end{tabular}

Table VII - Operation Performed in 24 Patients with Noncalculous Surgical Jaundice

\begin{tabular}{|l|c|c|c|}
\hline Operations & No and \% & Deaths & Mortality \% \\
\hline Cholecystojejunostomy and JJ with or without GJ & $14(58.3)$ & 2 & $14.2 \%$ \\
Laparotomy & $4(16.6 \%)$ & 1 & $25 \%$ \\
Hepaticojejunostomy & $2(8.3 \%)$ & - & - \\
Cholecystectomy with cystojejunostomy(Roux-en - Y) & $1(4.1 \%)$ & - & - \\
Ante colic GJ & $1(4.1 \%)$ & - & - \\
Whipple's procedure (Pylorus preserving) & $1(4.1 \%)$ & - & - \\
Drainage of pericolic abscess & $1(4.1 \%)$ & - & - \\
\hline Total & 24 & 3 & $12.5 \%$ \\
\hline
\end{tabular}




\section{Discussion}

Surgical jaundice due to noncalculous causes includes certain disease, which are not selflimiting in contrast to certain other forms of jaundice like hepatocellular and intrahepatic cholestatic jaundice. Prolonged period of obstruction to bile duct may lead to cholangitis, hepatic abscess, hepatic cirrhosis and its complications. So in order to avoid complications and mortality, irrespective of the cause and condition of the patient, decompression of the obstruction, either by surgery or percutaneous or endoscopic method is mandatory. In malignant cases of noncalculous surgical jaundice if diagnosis and treatment is not done in earliest possible time the disease will spread and will be fatal. Preoperative confirmation of diagnosis of surgical jaundice is a must because surgery in a misdiagnosed patient of hepatocellular jaundice may be fatal. Calculous causes should be differentiated from noncalculous causes because type of surgery differs in both groups. Elevation of biochemical parameters, Ultrasonography and CT scan findings of hepatobiliary system are simple and reliable guides for diagnosis and institution of proper therapeutic measures.

In the present study the maximum number of cases $(28 \%)$ was in the $7^{\text {th }}$ decade of life (Table No. -1). The youngest patient was 15 yrs and the oldest was 72 yrs of age. In periampullary and pancreatic carcinoma also most common (37.5\%) age group was 7 decade of life. The mean age for carcinoma head of pancreas was 65 years in a study of 100 patients by Ferla and Murray 1987. Carcinoma gall bladder was found equally in $6^{\text {th }}$ $\mathrm{W} 8^{\text {th }}$ decade of life (33.3\%), which was the most common age group for this disease. According to Robbins 1981 carcinoma gall bladder is found more in $6^{\text {th }}$ and 7 decade of life. Among the malignancy cases the youngest patient was 25 yrs and oldest was $72 \mathrm{yrs}$ old. It shows that malignancy is not a disease of old age only.

There were 14 males and 11 females in the present series showing a male dominance as it was reported by Bolandi et al 1979. In periampullary and pancreatic carcinoma male dominance was seen (M:F ratio 1.6:1) but in carcinoma gall bladder female dominance was seen (F:M ratio $2: 1)$. For carcinoma head of pancreas and periampullary region male:female was 1.8:1 according to G.La Ferla and WR Murray 1987. According to William, Cubilla and Maclean, 1979 M:F ratio for periampullary carcinoma was 4.5:1. In the literature higher female to male ratio (3:1) has been mentioned for carcinoma gall bladder (Moorhead and Warren 1976).

The cause of obstruction to extrahepatic bile duct was benign lesion in only one case (4\%) and the rest were malignant lesions (96\%). Periampullary and pancreatic carcinoma was the most common cause $(64 \%)$ in the present study. Other causes were carcinoma gall bladder (24\%), metastatic node at porta hepatis with primary at stomach or caecum (8\%) and choledochal cyst (4\%). Other previous workers Vickary et al 1979 and Toylor et al 1979 have also similar findings.

Shenker et al (1962) in 20 cases of obstructive jaundice found benign lesions (CBD stone) in 4 cases and malignant lesions in 16 cases. Carcinoma head of pancreas was the commonest and was detected in 10 cases $(50 \%)$ followed by carcinoma gall bladder in 2 cases, carcinoma in ampulla of Vater 2 cases, carcinoma bile duct in 1 case and carcinoma colon with enlarged node in porta hepatis in 1 case.

Madden, J.L. et al (1965) in their series of 140 cases of surgical jaundice found benign lesion in $71 \%$ and malignant lesions in $29 \%$ patients. Benign lesions were CBD stone $35.7 \%$, stenosis of papilla of Vater $12.1 \%$, chronic pancreatitis $7.8 \%$, CBD stricture $6.4 \%$, chronic cholangitis with stone $3.5 \%$, cholangiolitic hepatitis $2 \%$ and others like pancreatic cyst, CBD polyp, stone in cystic duct, cirrhotic nodule pressing porta each in one case (3.5\%). Malignant lesions were carcinoma pancreas $14.2 \%$, carcinoma gall bladder with gall stone $5.7 \%$, metastatic carcinoma $2.1 \%$ and carcinoma CBD with stone $1.4 \%$ of patients. 
Smith L(1979) in his series of 3527 bile duct obstruction found traumatic stricture as the leading cause of obstruction i.e. $44.1 \%$ cases. This is followed by neoplasm in $24.4 \%$, stone in $21 \%$, pancreatitis and its complications in $8 \%$, less common causes like sclerosing cholangitis, parasitic causes and lymphoma in $2.5 \%$ of cases. Out of the neoplastic causes carcinoma of pancreas and periampullary region were common and detected in $18 \%$ cases, followed by carcinoma bile duct $3.4 \%$, carcinoma gall bladder $0.8 \%$, metastatic carcinoma compressing porta hepatis $2.2 \%$.

Jaundice was the main presenting feature in all the patients in this series. The duration of icterus was $43.3 \pm 29.9$ days. The shortest duration was 7 days in a case of carcinoma head of pancreas and longest duration was 150 days in a case of periampullary carcinoma. Though the likelihood of a malignant obstruction is more in patient with prolonged period of jaundice, short duration at the time of presentation is not an exclusion in the diagnosis of malignancy. The prolonged period of jaundice at the time of presentation has been ascribed to the poor socio economical status and low health consciousness of the people, who report late for all their ailment. Type of jaundice was progressive in all cases except two cases of periampullary carcinoma where it was fluctuating, may be due to sloughing of tumor mass and transient relief of obstruction.

Among the other clinical features abdominal pain was commonest symptom seen in $76 \%$. The pain was usually constant dull aching type. Other commonly associated symptom was pruritus, found in $60 \%$ cases in the present study. The pruritus was attributed to retained bile acids in the blood. Earlier studies by various workers (Shenkar et al 1962, Kune 1972, Scharschmidt 1989) had similar reports.

Acholic stool was noticed in $44 \%$ of cases. This indicates complete obstruction of flow of bile into intestine.

Fever was the associated feature in $28 \%$ cases. It was due to cholangitis and in malignant cases it may be due to the increased metabolism by malignant process in the body.

Bleeding in the form of melaena was seen in one case of carcinoma stomach with secondaries at porta hepatis. This was due to bleeding from the primary tumour itself but there was possibility of bleeding from other sites also due to increased prothrombin time caused by deficit.

This higher value of serum bilirubin is corroborated to complete biliary obstruction in the presence of increased bilirubin production (e.g. increased red cell destruction in the tumor mass, ineffective erythropoiesis due to cachexin and folate deficiency) and hepatocellular dysfunction leading accelerated cholestasis due to metastasis to liver. Similar type of result was obtained by the previous workers. (O' Conner et al 1983, Scharschmidt 1989, Nundy et al 1989, Wig et al 1989).

The level of serum alkaline phosphatase was raised in all patients except two where it was in upper normal range. The mean value of serum alkaline phosphatase was $554.96 \pm 323.32 \mathrm{IU} / \mathrm{L}$. Normal serum alkaline phosphatase is $35-145$ IU/L. This elevated serum alkaline phosphatase is due to increased synthesis of the enzyme by the canalicular epithelium followed by regurgitation into the sinusoids.

The values of SGOT and SGPT found in this series were $113.88 \pm .90 .88 \mathrm{IU} / \mathrm{L}$ and 74.921 40.19 IU/L respectively. Usually in obstructive jaundice there is only mild elevation of transaminases. In the present series such high values of SGOT and SGPT were attributed to cholangitis and hepatocellular dysfunction due to secondaries in liver. Total serum protein was $6.92 \pm 0.48 \mathrm{mg} / \mathrm{dl}$. It was within normal limit in all patients except ' 3 patients where it was below normal. In urine urobilinogen was absent in all patients. Prothrombin time was prolonged in 5 patients which became normal after 24 hour of I.M injection of Vit-K. That indicates prolonged prothrombin time was due to deficiency of Vit-K as a result of lack of bile in the intestine. In rest of 
the patients prothrombin time was normal with mean value $13.28+1.4$ second.

Gates et al (1980) in their series found elevated SGOT/SGPT in 95\%, elevated alkaline phosphatase in $68 \%$ and prolonged prothrombin time in $16 \%$ cases.

All the 25 patients of the present series were subjected for ultrasonographic examination. In ultrasound dilated intrahepatic biliary radicles were seen all the 25 patients. Dilated bile duct was seen in $84 \%$ patients. Distended gall bladder was found in all the patients of periampullary or pancreatic carcinoma. Mass lesion in gall bladder was found in all the patients of carcinoma gall bladder. Pancreatic pathology in the form of SOL in Head of pancreas or periampullary area with or without dilated pancreatic duct was seen in patients of pancreatic and periampullary carcinoma. Hepatomegaly was seen in $76 \%$ patients.

Altogether 24 operations were done in 25 patients with surgical jaundice due to noncalculous causes of the present series. Out of them 3 patients died in the postoperative period before discharge from the hospital. The operations performed for malignant lesions were mostly biliary drainage procedure because of advanced stage of the disease with extensive metastasis. Tumour resection was done in only one case of periampullary carcinoma who presented earlier.

Cholecystojejunostomy with jejunojejunostomy was done in $14(58.3 \%)$ patients with periampullary or pancreatic carcinoma. A loop of jejunum, $45 \mathrm{~cm}$ below the duodenojejunal flexure was selected for anastomosis with gall bladder in front of the colon. Jejunojejunostomy was made between afferent and efferent loops of jejunum. Antecolic gastrojejunostomy was also made in two patients with duodenal obstruction and in another two cases as preventive measure for duodenal obstruction.

In periampullary carcinoma or carcinoma head of pancreas with obstructive jaundice palliative biliary bypass can be performed by cholecystojejunostomy or choledochoduodenostomy. Both a randomized prospective study and a collective review favour the use of the bile duct for better long-term results without associated increased perioperative morbidity or mortality (Sarfeh IJ. 1988, Watamapa P 1991). But cholecystojejunostomy is more frequently performed because it is technically easier.

In a review of more than 8000 patients reported in the English literature from 1965-1980, it was reported that the creation of a gastrojejunostomy did not increase the operative mortality rate. Moreover, if a gastrojejunostomy was not performed, $13 \%$ of patients had subsequent duodenal obstruction requiring a gastrojejunostomy before death, and nearly one in five of the remaining patients died with symptoms of duodenal obstructions. In patients requiring a second operation, mortality rate are higher. However, the ability of a gastojejunostomy to adequately palliate duodenal obstruction has been questioned.

Only laparotomy was done in $4(16.6 \%)$ patients. Definitive surgery was deferred because of extensive peritoneal involvement and adhesion of omentum. One of the patients having carcinoma head of pancreas died in the $9^{*}$ postoperative day due to hepatic encephalopathy. The remaining 3 patients had carcinoma gall bladder.

Hepaticojejunostomy was done in two $(8.3 \%)$ patients with carcinoma gall bladder with distal bile duct obstruction.

In patient with choledochal cyst cholecystectomy with Roux -en -Y cystojejunostomy was done. Ideally there should be excision of cyst with biliary-enteric anastomosis in this case. Excision of cyst could not be done in this case because of chance of injury to portal vein and lack of facility for vascular surgery in this hospital.

In patient with carcinoma caecum with pericolic absess and secondaries at porta hepatis only drainage of abscess was done. Even palliative bypass could not be done due to poor general condition of the patient. He developed faecal fistula in postoperative period, which was managed conservatively. 
In patient with carcinoma stomach with secondaries at porta hepatis antecolic Gastrojejunostomy was done.

Pylorus-preserving pancreaticoduodenectomy was done in one patient of periampullary carcinoma. Reconstruction was done by end-to-end pancreaticojejunostomy, end-to-side choledocho jejunostomy, end-to-side duodenojejunostomy and side-to-side jejunojejunostomy. There was mild bile leakage after operation, which subsided after conservative treatment. There was no significant delayed gastric emptying.

In a prospective randomized study by P.W Lin and Y-J Lin both PPPD and the Whipple procedure were associated with low mortality and operative morbidity rates. There was no significant difference between PPPD and Whipple resection in terms of operative mortality and morbidity, operating time, blood loss and blood transfusion. PPPD was associated with more frequent delayed gastric emptying.

\section{Follow up Study}

The patients who had undergone bypass procedures were symptomatically better after operation and jaundice decreased but there were few episodes of cholangitis. Two patients died 1 and 1 month after operation respectively. One had carcinoma gall bladder with metastasis and undergone hepaticojejunostomy. Death was due to hepatorenal syndrome. Another patient who had undergone CJ with JJ for periampullary tumour died $1 * / 2$ month after operation due to hepatorenal syndrome.

The patient of carcinoma caecum with pericolic abscess with secondaries at portal hepatis died due to septicemia on $20 *$ postoperative day.

The patient in which PPPD was done was asymptomatic with relief of jaundice till the completion of this study.

The patient of choledochal cyst was also free of symptoms and jaundice relieved after operation. There was one episode of cholangitis during the period of follow up, which subsided with antibiotics. Since in this patient only bypass procedure for the cyst was done, there is chance of repeated attacks of cholangitis and also cholangiocarcinoma. So she should be carefully observed.

\section{Conclusion}

- Twenty-five patients with surgical jaundice due to noncalculous causes have been studied over a period of two years.

- Diagnosis of surgical jaundice due to noncalculous causes has been done preoperatively with the help of clinical examination, biochemical investigation, ultrasonographic examination, and CT scanning.

- In 24 cases diagnosis was confirmed during operation and in one case it was confirmed by FNAC.

- Biochemical investigations done are estimation of serum bilirubin, serum alkaline phosphates SGOT, SGPT and total serum protein.

- The highest incidence of noncalculous surgical jaundice was noted in the seventh decade of life and male dominated.

- The highest incidence of periampullary and pancreatic carcinoma was also found in seventh decade of life with male dominance. incidence of carcinoma gall bladder was found in sixth and eighth decade of life with higher predilection for females.bPeriampullary and pancreatic carcinoma was the commonest cause (64\%) of noncalculous surgical jaundice. Other causes were carcinoma gall bladder, carcinoma stomach and carcinoma caecum with secondaries at porta hepatis and choledochal cyst.

- Jaundice was the main presenting features in all the patients in this series.

- Other clinical features like abdominal pain $(76 \%)$, pruritus $(60 \%)$ acholic stool $(44 \%)$, fever $(28 \%)$ bleeding $(4 \%)$, palpable gall bladder $(80 \%)$, hepatomegaly $(60 \%)$ and ascites (24\%) were associated.

- There was a rise of serum bilirubin (15.24 \pm 7.39 $\mathrm{mg} / \mathrm{dl}$ ), serum alkaline phosphatase (554.96 \pm $323.32 \mathrm{IU} / \mathrm{L})$, SGOT (113.88 $\pm 90.88 \mathrm{IU} / \mathrm{L})$ and SGPT $(74.92 \pm 40.19 \mathrm{IU} / \mathrm{L})$ in almost all cases. 
Total serum protein in most of the cases was within normal range. Prolonged prothrombin time correctable by parenteral Vit. - K. found in 5 cases (20\%).

- In ultrasonography dilated IHBR (100\%), dilated bile duct (84\%), Hepatomegaly $(76 \%)$, distended gall bladder $(64 \%)$ pancreatic pathology $(64 \%)$ and ascites (24\%) were noted.

-The operations performed were mostly bypass procedures like cholecystojejunostomy with jejunojejunostomy and gastrojejunostomy, hepaticojejunostomy and Roux-en-Y cystojejunostomy. One curative surgery done was pylorus-preserving pancreaticoduodenectomy without significant post operative complication. Post-operative mortality for malignant cases was $12.5 \%$. The patients in which bypass procedure was done and survived up to the completion of this study suffered recurrent attacks of cholangitis. Long term survival and results could not be assessed because of short period of study.

The present day approach to a patient with surgical jaundice is to establish the presence of an obstruction in the biliary tract and the level and nature of the lesion quickly and precisely. By meticulous analysis of clinical and biochemical parameters a surgeon can reach to a diagnosis in many cases. As diagnosis must be confirmed before taking the patient to operation theatre for operation, ultrasonographic demonstration of dilated biliary tree is a good criterion for differentiation from intrahepatic causes of jaundice. Ultrasound and CT scan can help to differentiate calculous causes from noncalculous causes of surgical jaundice. Cholecystojejunostomy with jejunojejunostomy properly performed is a good operation for palliative biliary-enteric decompression in case of malignant obstructive jaundice. The patient can have a better quality of life in spite of the presence of a dreaded disease during the short life span. In early cases of periampullary carcinoma pylorus-preserving pancreaticoduodenectomy can be done with good results. Thus the present study has been very helpful in deciding the plan of management in noncalculous surgical jaundice patients and to know their prognosis.

\section{References}

1. Agarwat S.K, Jirora S, (Physiological and biochemical changes in obstructive jaundice. Surgery Update 2000, p.p 21-25.

2. Jihrendt SA, pitt HA; (Biliary tract, In : Sabiston text book of Surgery Ed Townsend CM, 2001.

3. Jizuma Yoshikawa L Jiraida T Takasaki K Intraoperative evaluation of the depth of invasion of galbladder cancer, AM J Surg.. 1999; 178: 381-384.

4. Bachettier M Nakono H Ossouttzogtou E Weber JC, (Bouajema K WoLf P, JaecK $\mathrm{D}$ : Is pancreaticoduodenectomy with mesentericoportal venous resection safe and worthwhile? Jim J Surg, 182(2001)120-129.

5. Berg -dahC L. Qatt (Bladder carcinoma first diagnosed at macroscopic examination of gait bladders removed for presumed benign disease, Ann Surg 1980; 191:19-22.

6. Bismuth $\mathrm{K}$, Nakache $\mathrm{R}$ (Diamond $\mathrm{T}$. Management Strategies in resection for hilar cholangiocarcinoma. Jinn Surg 215:31,1992.

7. Buchter 31, Friess IL, Kempa I et at (Role of octreotide in the prevention of postoperative complications following pancreatic resection. Jim J Surg 1992; 163:125.

8. Casavitta FJi, Marsh JW, Iwatsuki S, et at: Hepatic resection and transplantation for peripheral cholangiocarcinoma. J Am cot Surg 195: 429,1997.

9. Chew DKW, Jittiyeh FF. Experience with the Whipple procedure (pancreaticoduodenectomy) in a university affiliated community Hospital Jim J Surg. 1997; 174:312-315.

10. Chitwood W. R. Meyers 1982. (Diagnosis $\&$ Treatment of -primary extra Hepatic 
Site duct tumors. Jim. J. Surg 143, 99 105.

11. Consentino C, Luck S, Raffensperger J, et at. Choledochal duct cyst: Resection with physiological reconstruction. Surgery 1992:112:740-8.

12. Cooperberg (P.L. - 1978. high resolution real time evaluation of the normal structure (Biliary tract, Radiology 129.477- 480.

13. Cotton (KB, (Perey 1964 - Exploration of CBD, Surg Gyn. \& Obst. 118: 20.

14. Cuberta fond P Matthonnet M, gainant A, Launosis B. Radical Surgery for gall bladder cancer. Results of the French surgicat association survey. hepatogastroenterology 1999; 46:1567-1571.

15. Dipatma A.Reggie 1979. The accuracy of $\mathrm{CT}$ in the screening of Obstructive Jaundice, (Diagnosis Imaging 48 (3): 142 $-148$.

16. Ferla g. La \& Murray 1987 - Ca of the head of pancreas (Br. J. Surg, 74: 212 213.

17. Fittin R, et at. 1978 Lipoprotein X \& diagnosis of cholestasis. Clinical Chim Acta; 85(1): 41-47.

18. Gates, g. F. Sinatra, F. R., Thomas, D. W. Cholestatic syndrome in infancy and childhood, AM 134:1141 -1148 (1980).

19. Gibson RN, Yeung E Thompson JN et at. (Bite duct obstruction: radiologic evaluation of Level, cause and tumour resectibility. Radiology 1986; 160: 43.

20. Gouma D.J, Van greenR.C.I, Van gulik TM. de Haan RJ, de Wit LT, Busch O.R.C, 06ertop H; Rates of complications and death after pancreaticoduodenectomy : Risk factors and the Impact of Hospital volume: Jinn Surg 232:6,786-795.

21. Grobmyer SR, Rivadeneira (EXE, goodman CA, Mackrell P, Lie6erman MD, Daly JM. (Pancreatic anastomotic failure after pancreaticoduodenectomy. Am J Surg. 2000; 180:117-120. 